\title{
Identification of novel biomarkers in chronic immune thrombocytopenia (ITP) by microarray-based serum protein profiling
}

\author{
Gürkan Bal, ${ }^{1}$ Matthias E. Futschik, ${ }^{2}$ \\ Daniela Hartl, ${ }^{3}$ Frauke Ringel, ${ }^{1}$ \\ Julian Kamhieh-Milz, ${ }^{1}$ Viktor Sterzer, ${ }^{1}$ \\ Jörg D. Hoheisel, ${ }^{4}$ Mohamed S. S. \\ Alhamdani $^{4, *}$ and Abdulgabar Salama ${ }^{1, *}$ \\ ${ }^{1}$ Institute for Transfusion Medicine, Charité \\ Universitätsmedizin, Berlin, Germany, ${ }^{2}$ Center \\ for Biomedical Research, Universidade do \\ Algarve, Faro, Portugal, ${ }^{3}$ Berlin-Brandenburg \\ Centre for Regenerative Therapies (BCRT), \\ Berlin, and ${ }^{4}$ Division of Functional Genome \\ Analysis, Deutsches Krebsforschungszentrum, \\ Heidelberg, Germany
}

Received 20 August 2015; accepted for publication 6 October 2015

Correspondence: Dr. Gürkan Bal, Institute for Transfusion Medicinem, Charité -

Universitätsmedizin Berlin, Augustenburger

Platz 1, 13353 Berlin, Germany.

E-mail: guerkan.bal@charite.de

*These authors contributed equally to this work.

\section{Summary}

The pathological mechanisms underlying the development of immune thrombocytopenia (ITP) are unclear and its diagnosis remains a process of exclusion. Currently, there are no known specific biomarkers for ITP to support differential diagnosis and treatment decisions. Profiling of serum proteins may be valuable for identifying such biomarkers. Sera from 46 patients with primary chronic ITP and 34 healthy blood donors were analysed using a microarray of 755 antibodies. We identified 161 differentially expressed proteins. In addition to oncoproteins and tumour-suppressor proteins, including apoptosis regulator BCL2, breast cancer type 1 susceptibility protein (BRCA1), Fanconi anaemia complementation group $\mathrm{C}$ (FANCC) and vascular endothelial growth factor A (VEGFA), we detected six anti-nuclear autoantibodies in a subset of ITP patients: anti-PCNA, anti-SmD, anti-Ro/SSA60, anti-Ro/SSA52, anti-La/SSB and anti-RNPC antibodies. This finding may provide a rational explanation for the association of ITP with malignancies and other autoimmune diseases. While RUNX1 mRNA expression in the peripheral blood mononuclear cells (PBMC) of patients was significantly downregulated, an accumulation of RUNX1 protein was observed in the platelets of ITP patients. This may indicate dysregulation of RUNX1 expression in PBMC and megakaryocytes and may lead to an imbalanced immune response and impaired thrombopoiesis. In conclusion, we provide novel insights into the pathogenic mechanisms of ITP that warrant further exploration.

Keywords: chronic autoimmune thrombocytopenic purpura, cancer, B cell CLL/lymphoma 2, runt-related transcription factor 3, thrombocytopenia.
Autoimmune thrombocytopenia (ITP) is a well-defined disorder that develops spontaneously and without any known causes (primary form). ITP also appears in association with other conditions, such as infections, malignancies, other autoimmune diseases and immunodeficiencies (secondary form) (Sullivan et al, 1997; Liebman \& Stasi, 2007; Rodeghiero et al, 2009; Stasi et al, 2009; Krauth et al, 2012). A number of genetic associations (Wu et al, 2005; Emmerich et al, 2007) have been identified and several well-known phenomena have been demonstrated to occur in ITP, such as autoantibodies (Harrington et al, 1951), T-cell mediated cytotoxicity (Olsson et al, 2003), complement activation (Cines \& Schreiber, 1979), deficiency of platelet production
(Ballem et al, 1987), loss of peripheral immune tolerance (Fogarty, 2011), dominant proinflammatory states (Guo et al, 2007), and various $\mathrm{B}$ and $\mathrm{T}$ lymphocyte dysfunctions (Olsson et al, 2005; Li et al, 2012). However, our understanding of ITP diagnosis, therapy, and pathogenesis remains limited. Its diagnosis is one of exclusion, and symptomatic treatment eases the symptoms without addressing the basic cause of the disease.

The pathogenic mechanisms of chronic refractory ITP (cITP) are complex and the initiating event remains elusive. Due to the complexity of the immune system and heterogeneity of the disease, it is difficult to elucidate all factors responsible for the development of cITP. Hitherto, only two 
candidate biomarkers have been reported in two studies to support differential diagnosis and treatment decisions in ITP. One of these studies, based on one-dimensional gel electrophoresis and the quantitation of identified proteins by liquid chromatography-tandem mass spectrometric analysis, found significantly higher levels of ceruloplasmin in patients with ITP compared with non-ITP patients and controls (Chousa et al, 2008). A second study used two-dimensional gel electrophoresis and found significantly lower haptoglobin levels in ITP patients non-responsive to splenectomy than in the responders (Zheng et al, 2012). However, the results of both studies are clinically less conclusive and are limited due to the techniques applied. Therefore, the present study applied antibody microarray to identify potential factors in the sera of patients and control individuals. Serum reflects the body's whole cellular metabolism and the release of proteins from a particular organ or a group of cells into the circulation due to physiological or pathophysiological alterations.

cITP patients may not only develop serious bleeding, but also other complications. The risk of haematological malignancies, such as acute myeloid leukaemia (AML), chronic lymphocytic leukaemia (CLL), Hodgkin lymphoma (HL), and non-Hodgkin lymphomas (NHL), has been reported to be significantly increased in cITP patients (Norgaard et al, 2011). On the contrary, immune-mediated platelet destruction is not uncommon in haematological malignancies (Krauth et al, 2012). This secondary ITP has been identified in some solid tumours and most lymphoproliferative disorders, but is more prevalent in patients with AML, CLL, HL and the myelodysplastic syndromes (MDS) (Cines et al, 2009; Liebman, 2009). This may be an indication of mutual pathophysiological mechanisms in cITP and haematological malignancies. A prediction of progression from cITP to malignancies would be useful.

Due to the aforementioned increased risk of haematological malignancies amongst patients with cITP, a set of oncoproteomic antibodies strogly related to haematological malignancies were selected for analysis. The present study should provide new insights in the pathophysiological mechanisms associated with haematological malignancies in cITP and therefore identify biomarkers that may improve differential diagnosis and support treatment decisions in ITP.

\section{Materials and methods}

\section{Study design}

CITP was diagnosed in accordance with the 2011 guidelines of the American Society of Hematology (Neunert et al, 2011). All patients were in chronic phase of the disease and had been under treatment in our centre for at least 1 year. Blood samples from 46 patients and 34 healthy controls were compared by proteomic profiling. Each patient was individually classified as being in active phase or remission phase according to their clinical parameters, such as platelet count, bleeding tendency, medication and prognosis. Active cITP was diagnosed in 34 patients, whereas 12 patients were in remission. Hereby, platelet counts were used as the main criteria for definition of remission $\left(>100 \times 10^{9} / 1\right)$ or active phase $\left(<100 \times 10^{9} / 1\right)$; bleeding tendency, altered platelet counts before and after the sampling date were also used as a decisive parameter to define the phase of disease as active or remission. During the course of the study, participating patients did not receive drugs known to induce thrombocytopenia. Anti-platelet autoantibodies were detected in 17 patients. Twenty patients were receiving immunosuppressive therapy with glucocorticoids and azathioprine. Among the remaining patients $(n=26)$, the spontaneous development of the disease was left untreated because they did not demonstrate an increased risk of severe bleeding; however, six of these patients were splenectomized. A summary of the study groups is provided in Table S1. Healthy control subjects $(n=34)$ were volunteer blood donors who received no drugs within $14 \mathrm{~d}$ prior to blood donation and had platelet counts greater than $250 \times 10^{9} / 1$. These values were measured before donation. This study was approved by the Ethical Institutional Review board of the Charite University Berlin, Germany (EA2_130_09).

\section{Further investigations}

Findings from protein microarray analyses were further investigated using enzyme-linked immunosorbent assay (ELISA), line immunoassay (LIA) and Western Blot assays. Due to a lack of material remaining after microarray analysis of the first cohort, we performed further analyses with new cohorts, which consisted partly of patients from the first cohort mixed with new samples. VEGFA ELISA assays were performed with 33 healthy controls and 46 patients with cITP (33 active phase, 13 remission). LIA assays were performed with 40 patients and 20 healthy controls.

\section{Serum separation}

Blood samples were collected in serum separating tubes (catalogue number 367955, Becton Dickinson, Heidelberg, Germany) that contained a gel to clearly separate serum from cellular components without any anticoagulation. All samples were processed under identical conditions. Sera were obtained by centrifugation at $1500 \mathrm{~g}$ for $10 \mathrm{~min}$. Protease inhibitor cocktail (Complete Mini EDTA-free, Roche, Mannheim, Germany) was used to inhibit protease activity. Samples were frozen at $-80^{\circ} \mathrm{C}$ until analysis.

\section{Microarray preparation}

Antibody microarrays were produced as previously described (Alhamdani et al, 2010, 2012; Schroder et al, 2010). Briefly, all antibodies were diluted to a final concentration of 
$1.0 \mathrm{mg} / \mathrm{ml}$ in spotting buffer containing $100 \mathrm{mmol} / \mathrm{l}$ sodium carbonate ( $\mathrm{pH} 8.5$ ), $5 \mathrm{mmol} / \mathrm{l} \mathrm{MgCl}_{2}, 0.01 \%$ (w/v) sodium azide, $0 \cdot 5 \%(\mathrm{w} / \mathrm{v})$ dextran sulfate and $137 \mathrm{mmol} / \mathrm{l}$ sodium chloride. Antibodies were spotted in quadruplicates on epoxysilane slides (Schott Nexterion, Jena, Germany) using a Microgrid microarraying robot (BioRobotics, Cambridge, UK) with SMP3B pins (Telechem, Sunnyvale, USA). Antibodies against $\beta$-actin (ACTB), human IgM, glyceraldehyde3-phosphate dehydrogenase and albumin were spotted as controls. Negative controls consisting of only spotting buffer were added, as well as additional control antibodies (e.g. antibodies directed against mouse immunoglobulin gamma). All controls were spotted in $8-18$ copies across the entire array to ensure a good distribution of controls for normalization purposes. After printing, the slides were maintained at $4^{\circ} \mathrm{C}$ and $50-60 \%$ humidity overnight. Printed slides were stored in dry conditions at $4^{\circ} \mathrm{C}$ until use.

\section{Sample labelling}

The labelling of serum proteins was performed as previously described (Alhamdani et al, 2010, 2012; Schroder et al, 2010). Briefly, serum samples were labelled at a $2 \cdot 0 \mathrm{mg} / \mathrm{ml}$ protein concentration with the $N$-hydroxysuccinimide esters of the fluorescence dyes Dy-549 or Dy-649 (Dyomics, Jena, Germany) at a dye/protein labelling ratio of 7.45 moles in $100 \mathrm{~mm}$ sodium carbonate buffer ( $\mathrm{pH} \mathrm{8.5)}$ for $2 \mathrm{~h}$ with constant shaking at $4^{\circ} \mathrm{C}$ in the dark. The labelling reaction was stopped by adding $10 \%(\mathrm{w} / \mathrm{v})$ glycine in sodium carbonate buffer $(100 \mathrm{~mm}, \mathrm{pH} 8.5)$ and continuous shaking at $4^{\circ} \mathrm{C}$ for an additional $20 \mathrm{~min}$ to quench the unreacted dye. Subsequently, complete protease inhibitor mixture tablets (Roche) were added as recommended by the manufacturer. Labelled samples were either incubated directly or stored as aliquots at $-20^{\circ} \mathrm{C}$.

\section{Protein array incubation}

Each sample was incubated competitively with a common reference that was made by a pool of all serum samples in a dual-colour mode as previously described (Alhamdani et al, 2012). Briefly, microarray slides were equilibrated to room temperature for $20 \mathrm{~min}$, transferred to Quadriperm chambers (Greiner Bio-One, Frickenhausen, Germany), and washed twice with phosphate-buffered saline (PBS) solution containing $0.05 \%(\mathrm{w} / \mathrm{v})$ Tween 20 for $5 \mathrm{~min}$ and $10 \mathrm{~min}$. Slides were blocked with $10 \%$ non-fat dry milk (Biorad, Munich, Germany) prepared in PBS with $0.05 \%$ Tween 20 for $3 \mathrm{~h}$ at room temperature in the dark. Sample incubation was performed in the same chambers using $50 \mu \mathrm{g}$ of labelled proteins in $5 \mathrm{ml}$ of PBS containing $0.05 \%$ Tween 20 and $10 \%$ non-fat dry milk overnight at $4^{\circ} \mathrm{C}$ in the dark. Finally, the slides were washed four times for $5 \mathrm{~min}$ each with PBS containing $0.05 \%$ Tween 20, rinsed thoroughly with distilled water, and dried in a ventilated oven at room temperature. Scanning of the slides was performed using a ScanArray 5000 unit (Perkin Elmer, Waltham, USA) set at the identical instrument laser power and photomultiplier intensity in each experiment. Spot segmentation was performed using GenePix Pro 6.0 (Molecular Devices, Sunnyvale, USA).

\section{Platelet isolation}

Platelet-rich plasma (PRP) was obtained from whole blood taken into citrate-anticoagulated vacutainers by centrifugation at $200 \mathrm{~g}$ for $20 \mathrm{~min}$ at room temperature without brake. The supernatant (PRP phase) was transferred into a new tube and HEP buffer $(140 \mathrm{mmol} / \mathrm{l} \mathrm{NaCl}, 2.7 \mathrm{mmol} / \mathrm{l} \mathrm{KCl}$, $3.8 \mathrm{mmol} / \mathrm{l}$ HEPES, $5 \mathrm{mmol} / \mathrm{l}$ EGTA, $\mathrm{pH} 7 \cdot 4$ ) at a $1: 1$ ratio $(\mathrm{v} / \mathrm{v})$ was added. After gently mixing, the samples were centrifuged at $100 \mathrm{~g}$ for $15 \mathrm{~min}$ at room temperature. Supernatants were transferred into a new tube and spun at $800 \mathrm{~g}$ for $15 \mathrm{~min}$ at room temperature to pellet the platelets. Pellets were washed once with PBS and then lysed in radioimmunoprecipitation assay (RIPA) buffer with proteinase inhibitors (Complete Mini EDTA-free, Roche, Germany) to produce platelet protein lysates.

\section{Immunoblotting of platelet RUNX1 and RUNX3 proteins}

Protein lysates of platelet samples from study patients and healthy controls were separated by sodium dodecyl sulphate polyacryamide gel electrophoresis and transferred to nitrocellulose membranes. The membranes were probed with antibodies directed against RUNX1 (D33G6 XP ${ }^{\circledR}$ Rabbit monoclonal antibody cat no: \#4336, Cell Signaling Technology, Danvers, MA, USA) and RUNX3 (D6E2 Rabbit mAb, Cell Signaling Technology). The corresponding band of each protein was detected by referenced molecular weight using a protein ladder.

\section{Separation of peripheral blood mononuclear cells (PBMC) and RNA isolation}

PBMC separation and RNA isolation were performed using samples from 12 cITP patients and 12 healthy controls. PBMC were isolated from $9 \mathrm{ml}$ of whole blood using a Ficoll gradient (Biochrom AG, Berlin, Germany). Isolated PBMC were lysed in Trizol (Invitrogen, Carlsbad, CA, USA) and afterwards RNA was extracted as previously described (Chomczynski \& Sacchi, 1987).

\section{Gene expression quantification}

cDNA was synthesized from $2 \mu \mathrm{g}$ of total RNA using the high capacity RNA-to-cDNA master mix (Applied Biosystems, Foster City, CA, USA) according to the manufacturer's protocol. The cDNAs were subjected to quantitative 
polymerase chain reaction (PCR) using SYBR Green Supermix (Applied Biosystems) and detected on the StepOnePlus ${ }^{\mathrm{TM}}$ Real-Time PCR System (Applied Biosystems) platform. For quantification, the $\Delta \mathrm{Ct}$ algorithm was applied using $A C T B$ as a housekeeping gene.

\section{Measurement of serum levels of VEGFA}

Vascular endothelial growth factor A (VEGFA) levels were measured using a specific ELISA (PromoKine Human VEGFA ELISA Kit, catalogue number: PK-EL-64406, PromoCell, Heidelberg, Germany) using $50 \mu \mathrm{l}$ of serum according to the manufacturer's recommendations. Serum levels of VEGFA were calculated from a standard curve generated using recombinant human VEGFA (PromoCell).

\section{Line immunoassay (LIA)}

The recomLine ANA/ENA IgG (Mikrogen, Neuried, Germany) is a LIA that detects the presence of autoantibodies against a variety of auto-antigens present in autoimmune diseases (RNP68, RNPA, RNPC, SmB, SmD, Ro/SSA60, Ro/ SSA52, La/SSB, Rib-P, PCNA, CENP-B, scl70, Jo-1, histone, and dsDNA). The test procedure was performed according to the manufacturer's instructions. Briefly, $20 \mu \mathrm{l}$ of serum was diluted at 1:100 and added on a test strip in an incubation tray for $1 \mathrm{~h}$. The test strip was then washed thrice and incubated with peroxidase conjugated anti-human antibodies (IgG-specific) for $45 \mathrm{~min}$. After repeated washes, $1.5 \mathrm{ml}$ of the substrate solution was added and incubated for $8 \mathrm{~min}$. The strip was the washed thrice with deionized water and the coloured bands that developed on the test strips were analysed.

\section{Bioinformatic analysis and statistics}

Using analytical methods analogous to those for DNA microarrays, the datasets were normalized using a quantile normalization approach. The protein expression of cITP and healthy controls was compared based on a linear model with a common reference. Comparisons of the differences in protein expression between the groups were derived using the empiric Bayes moderated $t$-test implemented in the linear models using the Bioconductor software microarray data (LIMMA) package (Smyth, 2004). P values were adjusted for multiple testing using the Benjamini-Hochberg method. Functional enrichment analysis of differentially expressed protein was performed by categorizing proteins within the annotations of the Gene Ontology (GO) using the Database for Annotation, Visualization and Integrated Discovery (DAVID, https://david.ncifcrf.gov/). Hereby, enrichment of the protein in the GO was calculated based on 679 distinct serum proteins spotted on our chips as a background dataset (Huang da et al, 2009).
The student's t-test and Mann-Whitney $U$ test were used to compare values of serum levels of VEGFA or RNA expression values of VEGFA, RUNX1 and RUNX3. A $P$ value less than 0.05 was considered significant. Pearson's correlation coefficient was calculated to examine the association between VEGFA levels and the platelet counts of patients.

\section{Results}

\section{Oncoproteomic profiling of serum samples}

We performed antibody microarray analysis on serum samples from 46 cITP patients and 34 healthy controls using 755 antibody probes corresponding to 679 distinct target proteins. In total, the expression of 165 probes, corresponding to 161 distinct proteins, was significantly different in the ITP group. To explore the biological significance of differentially expressed proteins, we examined their enrichment in the GO. Significant overrepresentation of proteins was identified in several interesting GO categories including 'blood coagulation' $(P=0 \cdot 01)$, 'induction of apoptosis' $(P=0.04)$, 'activation of caspase activity' $(p=0.04)$ and 'DNA damage response, signal transduction' $(P=0.03)$ (Table S2).

The expression of many oncoproteins and tumour-suppressor proteins, including HPV16 E1 protein-binding protein (TRIP13), breast cancer type 1 susceptibility protein (BRCA1), Fanconi anaemia complementation group C (FANCC), and breast carcinoma amplified sequence 1 (BCAS1), were significantly altered in the serum of cITP patients. The expression of 77 proteins was decreased in ITP patients including VEGFA, CDKN2A (P16INK4) and apoptosis regulator (BAX), whereas the expression of 84 proteins was increased including Ewing sarcoma breakpoint region 1 protein (EWSR1), zinc finger protein 593 (ZNF593), and regulator complex protein LAMTOR1 (C11orf59). Lists of the 20 proteins with the highest fold increase and decrease (adjusted $P$ value $<0.05$ ) in expression are presented in Tables I and II.

To focus on differences between the patients in active or remission phase versus the healthy control group, the microarray data of both patient groups were analysed separately. A total of 134 distinct proteins showed significant differences between the active cITP and the healthy group (Fig 1, Table S3). Only 10 of these proteins were also significantly altered in the remission group. Interestingly, only eight proteins, including B cell CLL/lymphoma 2 (BCL2), interferon gamma (IFNG), fibroblast growth factor 2 (FGF2) and cyclin-G1 (CCNG1), demonstrated active disease statespecific changes in their serum abundance of cITP groups (Fig 1). In hierarchical cluster analysis, 134 differentially expressed proteins and 80 samples were grouped according to their expression profiles. While most of the patients in 
Table I. List of 20 proteins with the largest decreases in expression in patients with cITP.

\begin{tabular}{llll}
\hline Symbol & Biological process & FC & adj.Pval \\
\hline VEGFA & Angiogenesis, differentiation & $-2 \cdot 15$ & $0 \cdot 010$ \\
CDKN2A & Cell cycle & $-1 \cdot 82$ & $0 \cdot 013$ \\
BAX & Apoptosis, host-virus interaction & $-1 \cdot 81$ & $0 \cdot 019$ \\
SELE & Cell adhesion & $-1 \cdot 74$ & $0 \cdot 003$ \\
PCNA & DNA damage, repair, and replication & $-1 \cdot 70$ & $0 \cdot 042$ \\
AIFM1 & Apoptosis & $-1 \cdot 66$ & $0 \cdot 045$ \\
TG & Thyroid hormones biosynthesis & $-1 \cdot 60$ & $0 \cdot 016$ \\
TFPI2 & Blood coagulation & $-1 \cdot 60$ & $0 \cdot 006$ \\
EIF3B & Protein biosynthesis & $-1 \cdot 49$ & $0 \cdot 000$ \\
ATP6V1F & Hydrogen ion transport & $-1 \cdot 33$ & $0 \cdot 000$ \\
IFITM2 & Antiviral defence, Innate immunity & $-1 \cdot 31$ & $0 \cdot 000$ \\
RP9 & Cognition, RNA splicing & $-1 \cdot 30$ & $0 \cdot 029$ \\
EEF1G & Protein biosynthesis & $-1 \cdot 27$ & $0 \cdot 000$ \\
GPX4 & Oxidoreductase & $-1 \cdot 27$ & $0 \cdot 002$ \\
RPS2 & Translation & $-1 \cdot 26$ & $0 \cdot 027$ \\
CASP10 & Apoptosis & $-1 \cdot 26$ & $0 \cdot 013$ \\
MOXD1 & Copper ion binding & $-1 \cdot 26$ & $0 \cdot 005$ \\
TPM2 & Muscle contraction, regulation & $-1 \cdot 26$ & $0 \cdot 045$ \\
& of ATPase activity & & \\
TGFBI & Proliferation, differentiation & $-1 \cdot 24$ & $0 \cdot 011$ \\
RUNX3 & Transcription regulation & $-1 \cdot 24$ & $0 \cdot 006$ \\
\hline
\end{tabular}

FC, fold change compared to healthy donors; adj.Pval., BenjaminiHochberg adjusted $P$-value.

active disease state and the healthy controls were assembled as two groups, patients in remission did not form a separate group but were found to rather distribute between healthy controls and active ITP (Fig 2).

\section{Analysis of VEGFA expression using ELISA and reverse transcription PCR (RT-PCR)}

In the antibody microarray data, VEGFA expression was mostly decreased in the cITP group $(-2 \cdot 15$-fold change, adjusted $P=0 \cdot 01$ ). To confirm this finding, we performed ELISA for VEGFA on serum samples of 46 patients with ITP and 33 healthy donors (Fig 3B). The serum levels of VEGFA in patients with active ITP (median, $196 \mathrm{pg} / \mathrm{ml}$ ) were significantly lower $(P=0.002)$ than in healthy controls (median, $648 \mathrm{pg} / \mathrm{ml})$. In the remission group $(n=13)$, the levels of VEGFA were found to be similar to those of the healthy control group (median, $579 \mathrm{pg} / \mathrm{ml}$ ). The correlation coefficient between VEGFA levels and platelet counts was significant $(r=0.36, P=0 \cdot 001)$. As platelets are the main contributors of serum VEGF and ITP patients have lower platelet counts per se, detection of VEGFA in protein level from serum or plasma samples may cause biological bias. Therefore, we performed quantitative gene expression analysis from PBMC of 12 active ITP patients (platelet count median, $34 \pm 33 \times$ $10^{9} / 1$ ) and 12 healthy donors (platelet counts greater than $\left.250 \times 10^{9} / 1\right)$. As shown in Fig 3 A, compared with healthy controls, VEGFA mRNA expression was significantly
Table II. List of the 20 proteins with the highest increases in expression in patients with cITP.

\begin{tabular}{llll}
\hline Symbol & Biological process & FC & adj.Pval \\
\hline EWSR1 $^{*}$ & Transcription regulation & $2 \cdot 39$ & $0 \cdot 000$ \\
ZNF53 $^{*}$ & Transcription regulation & $1 \cdot 70$ & $0 \cdot 003$ \\
EWSR1 $^{*}$ & Transcription regulation & $1 \cdot 49$ & $0 \cdot 000$ \\
LAMTOR1 & Cell growth regulation & $1 \cdot 39$ & $0 \cdot 025$ \\
RPL10A & Ribosomal protein & $1 \cdot 36$ & $0 \cdot 020$ \\
HSPA1L & Nucleotide binding, stress protection & $1 \cdot 35$ & $0 \cdot 005$ \\
NR4A1 & Transcription regulation & $1 \cdot 34$ & $0 \cdot 020$ \\
SLC7A5 & Amino acid transport & $1 \cdot 33$ & $0 \cdot 000$ \\
NPY4R & G protein coupled receptor & $1 \cdot 32$ & $0 \cdot 017$ \\
& $\quad$ signalling pathway & & \\
FUBP1 & RNA binding & $1 \cdot 32$ & $0 \cdot 000$ \\
CTSD & Intracellular protein breakdown & $1 \cdot 28$ & $0 \cdot 021$ \\
HNRNPR & RNA binding & $1 \cdot 28$ & 0.009 \\
BRPF3 & Zinc ion binding & $1 \cdot 27$ & $0 \cdot 004$ \\
SREBF1 & Transcription regulation & $1 \cdot 26$ & $0 \cdot 000$ \\
GRB10 & Insulin receptor signalling pathway & $1 \cdot 25$ & $0 \cdot 000$ \\
BRCA1 & DNA repair & $1 \cdot 25$ & $0 \cdot 007$ \\
THBS3 & Cell adhesion & $1 \cdot 25$ & $0 \cdot 010$ \\
DDIT3 & Apoptosis, transcription regulation & $1 \cdot 24$ & $0 \cdot 009$ \\
PGF & Angiogenesis, differentiation & $1 \cdot 24$ & $0 \cdot 000$ \\
HOXC11 & Transcription regulation & $1 \cdot 24$ & 0.005 \\
\hline
\end{tabular}

FC, fold change compared to healthy donors; adj.Pval, BenjaminiHochberg adjusted $P$-value.

*EWSR1 antibodies were redundantly spotted on microarray chips.

decreased in the PBMC of cITP patients $(P=0.001$, mean cITP, $0.042 \pm 0.007$ vs. mean healthy donors, $0 \cdot 13 \pm 0.02$ ).

\section{Detection of anti-PCNA autoantibody using LIA}

Proliferating cell nuclear antigen (PCNA) exhibited one of the strongest changes in expression in the antibody microarray experiment (-1.7-fold change, adjusted $P$ value $=0 \cdot 04)$. As several reports have indicated that systemic autoimmune diseases with anti-PCNA autoantibody have a high frequency of thrombocytopenia (Yamanaka \& Takasaki, 1993) and many nuclear proteins exhibited lower expression levels in the microarray experiment, we analysed a panel of autoantibodies closely related to autoimmunity that could interfere with the antibody microarray experiments. Sera from 20 controls and 40 patients were tested for the following autoantibodies: PCNA, RNP68, RNPA, RNPC, SmB, SmD, Ro/SSA60, Ro/SSA52 (TRIM21), La/ SSB, Rib-, CENP-B, scl70, Jo-1, histone, and dsDNA. Autoantibodies against PCNA were detected in only 2 of 40 samples from cITP patients. Similarly, anti-RNPC, anti-Ro/ SSA52 (TRIM21) and La/SSB antibodies were each detected in 2 patients, whereas none of the 20 healthy control sera were positive for these autoantibodies. Anti-SmD and antiRo/SSA60 autoantibodies were each detected in only one patient and were not present in the control group (Table III). Altogether, 9 out of 40 cITP patients were 


\begin{abstract}
"Total ITP versus HD" and "active ITP versus HD" AP4B1, ARID4A, BAK1, BCAS1, BCL2A1, BRAF, BRCA1, BRPF3, LAMTOR1, C16orf58, C2, CACNA1G, CASP10, CD59, CDK1, CDK4, CDKN2B, CLDN4, CSF2RB, CTRB1, CTSB, CTSD, CTTN, DDIT3, EDNRB, EEF1G, EIF3B, EIF31, ENO2, EP300, F3, FANCC, FN1, FOS, GATA4, GPX4, GRIN2C, GRP, GSS, HNRNPR, HOXC11, HSPA1L, IFITM2, IFNGR1, IGF2, IL2, IL6R, ISOC1, KIAA0391, KLK11, LCP1, LY6K, MAPK1, MLH1, MMP12, MMP2, MOXD1, MSR1, MTA1, MTHFR, MUC3A, NCOR2, NFKB2, NR4A1, CDKN2A, PAPOLA, PDCD2, PDIA6, PIR, PKM, PLAU, PLAUR, PLK2, POU2F1, NPY4R, PRDM1, PSMB4, PTEN, PTPRCAP, RP9, RPL10A, RPS2, RPSA, RUNX3, RWDD2A, S100A8, SELE, SFN, SGCA, SGSM3, SLC16A3, SLC19A1, SLC35A2, SORBS3, SYK, SYNGR2, TAT, TFPI2, TG, TGFB2, TGFBI, THBS2, THBS3, THOC1, TJP2, TM4SF4, TMEM54, TMOD3, TNFRSF10B, TRAF4, UBXN4, VEGFA, VIL1, WNT2B, ZKSCAN1, ZNF593,
\end{abstract}

\section{"Total ITP versus HD"}

AIFM1, ALB, BAX, CADM1, CCNA2, CDKN2A, CFLAR, CYCS, CYP3A5, DKK2, ERBB2, ETS2, FLT1, GARS, IGHA1, IL6, LUM, MMP11, MMP7, MS4A2, MX2, NPY1R, PCNA, PYCR1, SDHB, SERPINE1, SP1, STK19, TIA1, TIMP1, TIMP3, TNF, TNFSF14, TPM2, ZBTB22

\section{"Total ITP versus HD", "active ITP versus HD" and "remission ITP versus HD" AKR1C3, ATP6V1F, EWSR1, FUBP1, GRB10, MXI1, PGF, SLC7A5, SREBF1, TRIP13}

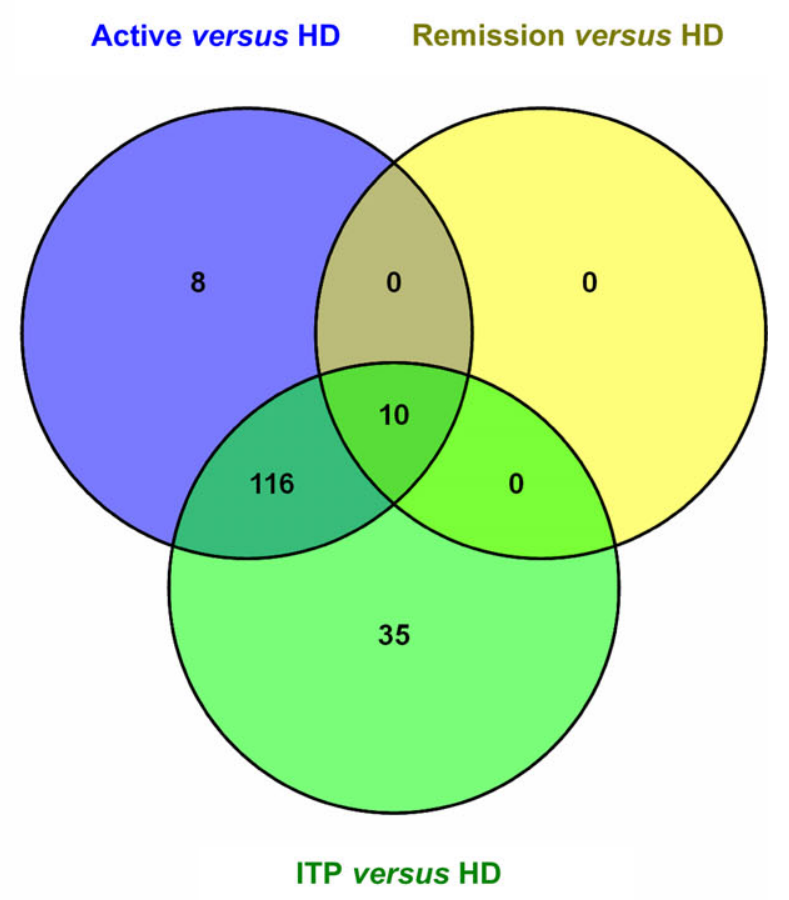

"Active ITP versus HD"
BCL2, CCNG1, FGF2, ICAM2, IFIT2, IFNG, MMP10,
NME2

Fig 1. Comparative Venn analysis of differentially expressed proteins. Protein sets from separately analysed groups according to their disease states and protein sets from unseparated analyses are compared. (ITP versus HD: protein set from unseparated analysis of all cITP patients versus healthy controls; active versus HD: protein set from active chronic ITP group separately analysed versus healthy controls; remission versus HD: protein set from chronic ITP patients in remission separately analysed versus healthy controls). ITP, immune thrombocytopenia; HD, healthy donor.

positive for anti-nuclear antibodies, whereas none of the 20 controls exhibited any reactivity with the nuclear antigens studied.

Analysis of RUNX3 and RUNX1 expression in platelets of cITP patients and healthy controls

Runt-related transcription factor 3 (RUNX3) expression was significantly decreased in the antibody microarray experiments (adjusted $P$ value $=0 \cdot 006$ ). To validate this observation independently of changes in platelet counts among the patient groups, we analysed RUNX3 protein expression in platelet lysates of cITP patients and healthy controls using Western blotting. As the RUNX family is a known regulator of megakaryopoiesis, we additionally analysed the expression of another member of the RUNX family, RUNX1, that was not included in the antibody microarray. Western blot analysis demonstrated decreased expression of RUNX3 in the platelets of $50 \%$ of patients with refractory immune thrombocytopenia (Fig 4). In contrast, an accumulation of
RUNX1 protein was detected in the platelets of most patients with cITP and healthy controls (Fig 5).

RUNX3 and RUNX1 mRNA expression in PBMC of cITP patients and healthy controls

Serum is comprised of proteins released from blood cells and tissue. To determine if the results of the microarray tests can be transferred to PBMC, we analysed RUNX3 and RUNX1 mRNA expression in the PBMC of cITP patients and healthy controls using real time PCR. As shown in Fig 3A, although RUNX3 mRNA expression was decreased in the PBMC of cITP patients relative to internal ACTB expression $(0 \cdot 804 \pm 0 \cdot 155$ vs. $1 \cdot 041 \pm 0 \cdot 102$, cITP and controls, respectively), the difference between both groups was not statistically significant $(P=0 \cdot 111)$. In contrast, RUNX1 analyses demonstrated a statistically significant decreased mRNA expression in PBMC of cITP patients compared with healthy controls $(P=0.001$, mean $0.243 \pm 0.033$ vs. $0.651 \pm 0 \cdot 113$, cITP and controls, respectively; Fig 3A). 


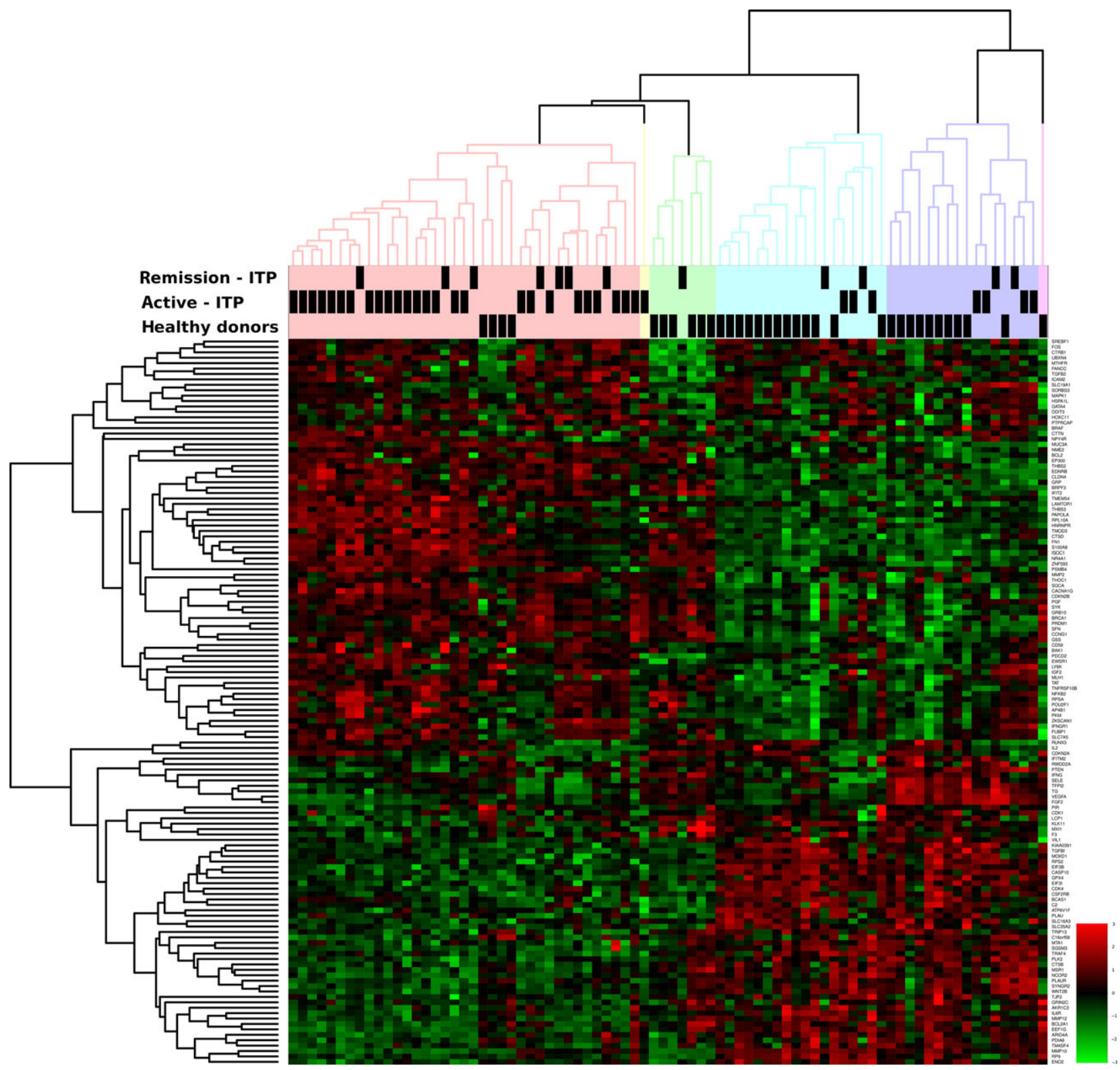

Fig 2. Heat map demonstrating unsupervised hierarchical clustering pattern of significantly differential expressed proteins. While samples from active cITP patients and healthy controls built two distinct cluster patterns, the samples from cITP patients in remission are distributed amongst both of these two clusters. Each row represents a single protein, and each column represents a single patient. Red represents increase expression; green, decreased expression. The disease state of each sample was defined as either active (platelet count $\left.<100 \times 10^{9} / 1\right)$, remission $($ platelet count $>100 \times 10^{9} / \mathrm{l}$ ) or healthy (platelet count $>250 \times 10^{9} / \mathrm{l}$ ). cITP, chronic immune thrombocytopenia.

\section{Discussion}

Genome- and proteome-wide measurements of disease-specific alterations may help to obtain a more comprehensive understanding of diseases. In addition to analysing DNA aberrations and RNA expression (Sood et al, 2008; Makroo et al, 2011), which may occasionally be useful for characterizing diseases, systematic profiling of proteins is the method of choice to ultimately identify principal components responsible for the various in vivo physiological and pathological processes. In our opinion, profiling of serum proteins (proteomics) is currently the most promising method for disease diagnosis and therapeutics for ITP and other autoimmune diseases. Two-dimensional gel electrophoresis and mass spectrometry-based comparative proteomic approaches have been widely used to discover biomarkers; however, these approaches have some limitations, particularly in terms of sensitivity, reproducibility, and resolution (Wittmann-Liebold et al, 2006; Han et al, 2008). To overcome this limitation, we preferred to apply the affinity-based proteomics approach 


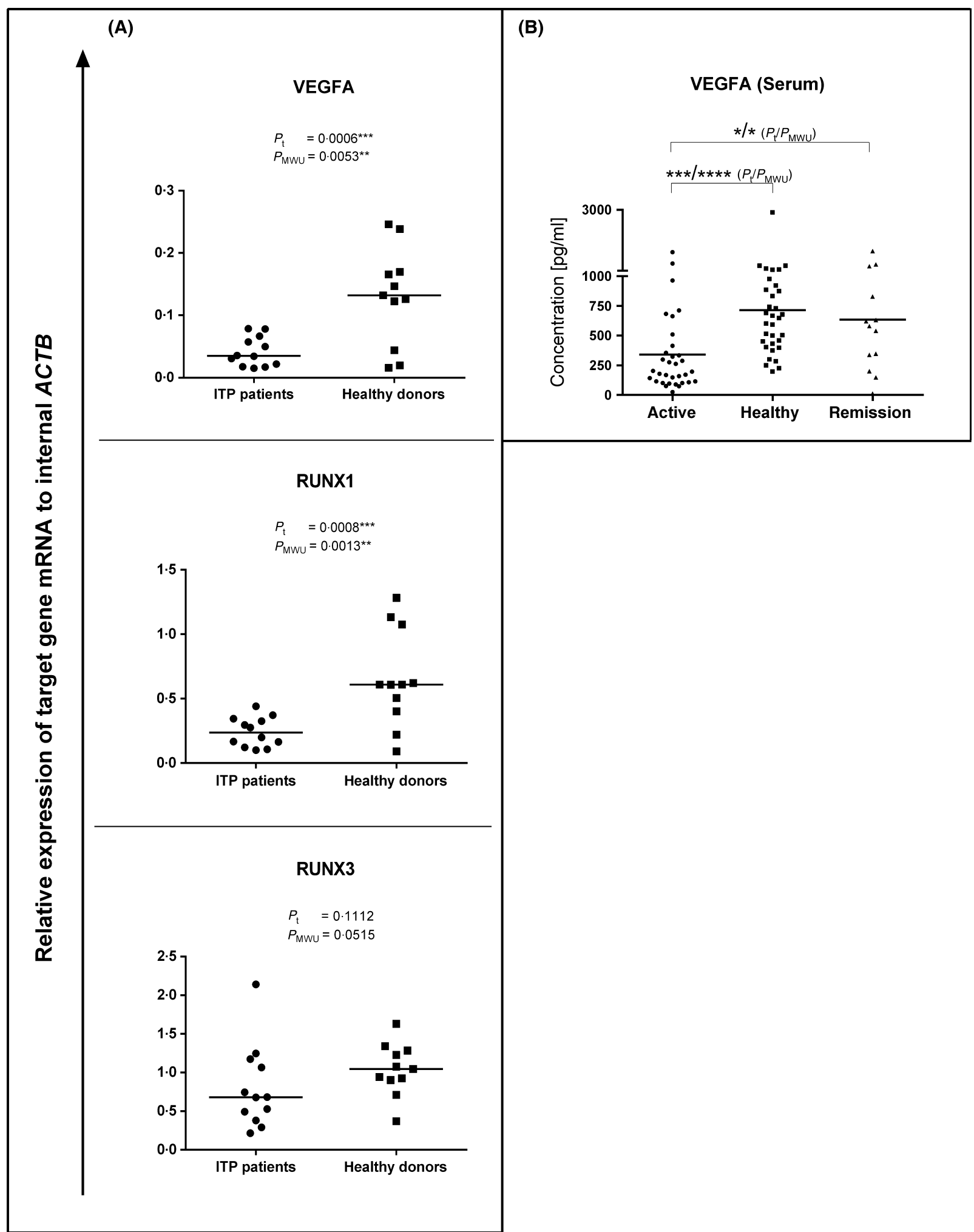

Fig 3. Expression of VEGFA, RUNX1 and RUNX3. (A) Relative expression of VEGFA, RUNX1 and RUNX3 mRNA in PBMC of active cITP patients $(n=12)$ and healthy controls $(n=12)$. Results are shown as expression of the target genes in comparison to internal expression of the housekeeping gene ACTB. (B) Serum protein concentration of vascular endothelial growth factor (VEGFA). The sera from 33 healthy controls and 46 patients with cITP, composed of 33 patients with active disease and 13 patients in remission, were analysed by ELISA for the presence of VEGFA. $\quad \mathrm{p}_{\mathrm{t}}, \quad P$-value from student $t$-test; $\mathrm{p}_{\mathrm{MWU}}, \quad P$-value from $\quad$ Mann-Whitney- $U$-test; $\quad * 0.01 \leq P<0.05 ; \quad * * 0.001 \leq P<0.01$; $* * * 0.0001 \leq P<0.001 ; * * * * P<0.0001$. 
Table III. Results of the analysis of sera from 20 healthy donors and 40 patients with cITP tested for the presence of fifteen nuclear autoantibodies. Only those patients with detectable autoantibodies are shown. There were no patients or healthy donors expressing antibodies against RNP68, RNPA, SmB, Rib-P, CEBP-B, scl70, jo-1, Histon or dsDNA.

\begin{tabular}{lllllll}
\hline Patient & RNPC & SmD & Ro/SSA60 & Ro/SSA52 & La/SSB & PCNA \\
\hline 1 & + & - & - & - & - & - \\
2 & - & - & - & - & - & - \\
3 & - & - & - & - & + & - \\
4 & - & - & - & - & + & - \\
5 & - & - & + & + & - & - \\
6 & + & - & - & - & - & - \\
7 & - & - & - & - & - & + \\
8 & - & - & - & - & - & + \\
9 & - & + & - & - & - & - \\
\hline
\end{tabular}

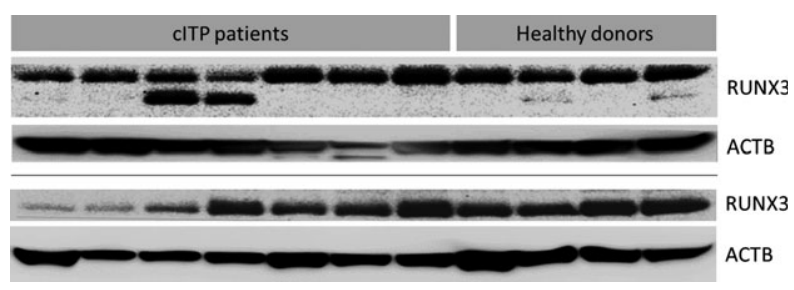

Fig 4. Protein expression of RUNX3 in platelets of cITP patients $(n=14)$ versus healthy controls $(n=8)$ were analysed using Western blot assays (ACTB as loading control). cITP, chronic immune thrombocytopenia

(antibody-based microarray) that is frequently applied in cancer research and diagnostics because of the sensitivity of the technique and its similarity to routinely applied affinity assay formats (Alhamdani et al, 2009; Hoheisel et al, 2013).

Protein biomarkers have improved the management of different diseases (Parker \& Borchers, 2014). Focusing on ITP, we sought to determine whether particular proteins are involved in both ITP and malignancies. In fact, a bidirectional association between ITP and malignancies has been reported; ITP occurs in patients with malignancies and cITP patients may develop haematological disorders (Liebman, 2009; Norgaard et al, 2011; Krauth et al, 2012). Using the antibody microarray described in this study, 165 antibodies yielded significantly different signal intensities, corresponding to 161 distinct proteins that showed significantly different expression in cITP. In agreement with the association of ITP and malignancies, many oncoproteins and tumour-suppressor proteins exhibited significantly different expression in cITP, including TRIP13, BRCA1, FANCC, BCL2 and BCAS1.

To date, numerous studies have indicated that the aetiology and disease status of ITP is closely related with an impaired balance of $\mathrm{T}$ helper cytokines (Th1/Th2) and $\mathrm{T}$ regulatory cell cytokines (Th3), but the reported data are mostly controversial (Ogawara et al, 2003; Ma et al, 2008) and some are contradictory (Panitsas et al, 2004; Ma et al, 2014). Similar to the finding in the most recent study (Ma et al, 2014),

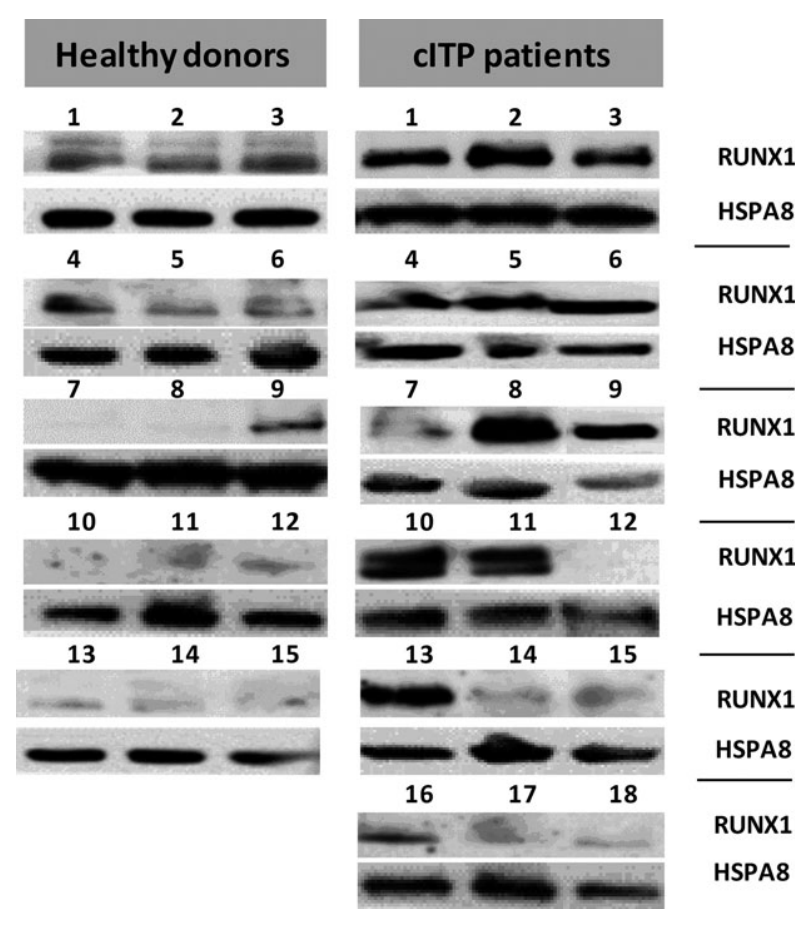

Fig 5. RUNX1 protein expression in platelets from patients with cITP $(n=18)$ versus healthy controls $(n=15)$ was analysed using Western blot assays (HSPA8 is the loading control).

we observed a significantly downregulated protein concentration of Th1 cytokines, interleukin (IL)2, IL6 and IFNG (only in active disease states) in the serum of cITP patients. Discrepancy with some of the studies might be due to the heterogenetic aetiology of ITP or the nature of the exclusion diagnosis in ITP. These were sustained by hierarchical cluster analysis of our antibody microarray data, where some of the ITP patients were distributed independently from their disease state between healthy controls and active states. In contrast to Th1 cytokines, the Th3 cytokine, transforming growth factor- $\beta 1$ (TGFB1), which is associated with an immunosuppressive profile, were consistently reported by several studies downregulated in ITP (Andersson et al, 2000, 2002). A significantly decreased TGFB1 concentration was also detected in the serum of patients using our antibody microarray. Consistent with this finding, it has been reported that decreased TGFB1 is correlated with deficient frequency and function of $\mathrm{CD}^{+} \mathrm{CD} 25^{+} \mathrm{FOXP} 3^{+}$regulatory $\mathrm{T}$ cells in patients with immune thrombocytopenia (Fahim \& Monir, 2006; Arandi et al, 2014).

Separate analysis of patients with active and remission states yielded significant differences of 134 distinct proteins in the active cITP group, whereas 126 of these were differentially expressed in both groups when combined. The detection of 126 mutual proteins indicates an accurate processing of samples and robustness of the antibody microarray data. However, differences in the expression levels of some proteins may occur between the individual disease states, active or remission. Interestingly, 8 distinct proteins were 
significantly differentially expressed only in the active disease state and comprised of FGF2, MMP10, IFNG, IFIT2, ICAM2, NME2, CCNG1 and BCL2 (Fig 1). Consistent with our results, the apoptosis regulator BCL2 was found to be upregulated in sera from children and adult cITP patients (Liu et al, 2005; Stasi et al, 2007). In agreement with other studies (Olsson et al, 2005; Chang-Lin et al, 2007), our results indicate that impaired apoptosis in patients with active ITP could be involved in active platelet destruction. Therefore, specific inhibition of BCL2 activity might achieve remission of ITP.

To evaluate the reliability of microarray analysis, we confirmed the results of the VEGFA concentration by ELISA. Microarray data were validated even with an alternative cohort, thus indicating a robust finding. Similar to the decreased levels of VEGFA identified with the antibody microarray, serum levels of VEGFA in patients with active ITP were significantly decreased (median, $196 \mathrm{pg} / \mathrm{ml}$ ) compared with healthy controls (median, $648 \mathrm{pg} / \mathrm{ml}$ ) by ELISA. Moreover, a significant correlation was observed between VEGFA levels and platelet counts $(r=0.36, \quad P=0.001)$. Although the ELISA demonstrated the reliability of the antibody microarray experiments, it is unclear whether VEGFA plays a role in the pathophysiology of cITP. The decreased concentration and positive correlation of VEGFA may be explained first by the lower platelet counts in patients with cITP and large amounts of VEGFA in platelet granules (Gunsilius et al, 1999). However, independent of platelet counts, significant changes in VEGFA expression in peripheral blood-derived $\mathrm{T}$ cells of patients with cITP have been detected at the transcriptional level (Jernas et al, 2013). Consistent with this finding, downregulated VEGFA mRNA levels were also detected in the PBMC of 12 cITP patients versus 12 healthy donors. In addition, increased soluble FLT1 concentrations $(P<0.05)$, a receptor for VEGFA, were detected in the serum of patients with CITP using the antibody microarray. Increased levels of soluble FLT1 may interfere with VEGFA and FLT1 interaction on megakaryocytes and may significantly inhibit megakaryocytic polyploidization (Casella et al, 2003), which is correlated with cITP development (Gerritsma et al, 1994). Another ligand of FLT1 is placental growth factor (PGF) and its expression was significantly increased (adjusted $P$-value $<10^{-16}$ ) in the sera of patients with cITP. Altogether, the altered concentrations of VEGFA, PGF and soluble FLT1 in patient groups indicate that a deregulated VEGF signalling pathway contributes to the pathophysiology of cITP. In agreement with this finding, increased PGF concentrations were used as a biomarker by Young et al (2010) to distinguish patients with ITP from patients with thrombocytopenia resulting from HELLP (haemolysis, elevated liver enzymes, and low platelets) syndrome during pregnancy.

A potential role of the RUNX family in the development of chronic autoimmune diseases, such as systemic lupus erythematosus (SLE), rheumatoid arthritis (RA) and psoriasis, has been reported by several authors (Helms et al, 2003; Tokuhiro et al, 2003; Alarcon-Riquelme, 2004). RUNX1 and RUNX3 have primarily been found to regulate genes expressed in the myeloid and lymphoid lineages (Otto et al, 2003). The targets of RUNX1 include B-cell specific tyrosine kinase (BLK), the T-cell antigen receptor (TCR) $\alpha, \beta, \gamma$ and $\delta$ chains, $\mathrm{CD} 3, \mathrm{CD} 4$, and granulocyte/macrophage colony stimulating factor (GM-CSF) in lymphoid cells (Otto et al, 2003). Moreover, RUNX3 and RUNX1 are required for CD8 $\mathrm{T}$ cell development during thymopoiesis (Woolf et al, 2003), and the regulatory $\mathrm{T}$ cell function is controlled by the interaction between FOXP3 and RUNX1 (Ono et al, 2007). A variety of abnormalities within the $\mathrm{T}$ cell population in ITP have been described (Semple \& Freedman, 1991; GarciaSuarez et al, 1993; Semple et al, 1996), including increased autoreactive $\mathrm{T}$ cell reactivity and decreased regulatory $\mathrm{T}$ cell function (Kuwana et al, 1998). We hereby present a statistically significant downregulation of RUNX1 mRNA expression in the PBMC of cITP that may lead to the variety of $\mathrm{T}$ cell abnormalities observed in cITP. A similar, but not statistically significant, downregulation of RUNX3 mRNA expression in the PBMC of most cITP was observed. Furthermore, we provide evidence for deregulated RUNX3 and RUNX1 appearance in platelets of cITP patients. In particular, RUNX1 accumulation in the platelet cytoplasm may indicate disturbed nuclear transport mechanisms in megakaryocytes in cITP patients. In addition to the immune-mediated destruction of platelets, megakaryocytic abnormalities, such as altered megakaryocytic maturation, are suggested to be one of the main pathogenic abnormalities in CITP (Gerritsma et al, 1994; Ucar et al, 2003). In this respect, the disturbed nuclear transport of RUNX1 may result in inadequate thrombopoiesis through aberrant expression of the thrombopoietin receptor, MPL, in megakaryocytes. Indeed, MPL in myeloid lineage cells and megakaryocytes is controlled by RUNX1 (Satoh et al, 2008; Yu et al, 2012). Moreover, RUNX1 is a key regulator for the switch of mitosis to endomitosis in megakaryocytes, linking polyploidization and megakaryocyte differentiation (Lordier et al, 2012).

Several authors have reported that a subpopulation of patients with ITP has autoantibodies to nuclear proteins and is at risk for developing SLE (Balsalobre Aznar et al, 1999; Unal et al, 2005; Hazzan et al, 2006). Conversely, anti-platelet antibodies have been detected in $78 \%$ of SLE patients, often without accompanied thrombocytopenia, and in up to $16 \%$ of these patients, isolated thrombocytopenia was the initial clinical manifestation (Yehudai et al, 2013). The differential diagnosis of cITP and secondary ITP in cases of SLE has prognostic implications. One of the potential prognostic factors, PCNA, had significantly decreased expression in the cITP group. To confirm this finding, we investigated a panel of autoantibodies; anti-PCNA is closely related to systemic autoimmunity and could have interfered with our antibody microarray experiments. However, anti-PCNA autoantibody was detected in only 2 of 40 cITP patients using LIA. These 
two patients were also included in the microarray analysis. In addition, we detected the anti-RNPC, anti-Ro/SSA52 and anti-La/SSB autoantibodies in two different cases. Altogether, 9 of 40 patients with cITP were positive for anti-nuclear antibodies, whereas none of the 20 control sera exhibited any reactivity with the nuclear antigens studied. As ITP may occur as a first manifestation of other immune systemic diseases, screening these factors in patients with ITP may be useful for the early recognition of patients at risk for progression to other diseases, facilitating the choice of therapeutic procedures (Adachi et al, 1990; Balsalobre Aznar et al, 1999). On the other hand, the presence of autoantibodies in the serum of ITP patients may have a great impact on the outcome of antibody microarrays. Autoantibodies in the serum of a patient may cause sequestration of their target proteins in vivo. More importantly, autoantibodies in serum samples may cause depletion of their targeted proteins from the protein pool (as a common protein reference, described in Material and methods) on each microarray chip. We detected anti-PCNA serum reactivity in two patients in our collective patient group, leading to the conclusion that the significantly downregulated PCNA protein expression detected in our microarray is not representative. Although this would be a technical bias, a controversial interpretation of downregulated proteins similar to our experience with anti-PCNA might help to detect novel autoantibodies in ITP.

The cyclin-dependent kinase (CDK) inhibitor gene CDKN2A (P16INK4) is an important tumour suppressor gene, which controls the checkpoint of the cell cycle at the G1/S phase transition and has a pivotal function in the induction of apoptosis and senescence, as well as quiescence, of cell cycles (Nobori et al, 1994; Stone et al, 1995). A senescence-like process was also detected in the physiological post-mitotic megakaryocytes, which is a contribution of CDK inhibitors induced by thrombopoietin (Besancenot et al, 2010). Moreover, CDKN2A has been reported to affect the expression of inflammatory cytokines from activated macrophages (Taniguchi et al, 1999; Murakami et al, 2012). We observed significantly decreased CDKN2A protein expression in the serum of a cITP patient using antibody microarray $(-0 \cdot 87$-fold change, adjusted $P$-value $=0 \cdot 01)$. Accordingly, increased expression of BMI1 protein, a transcriptional repressor of CDKN2A, was recently reported in the autoreactive T-cells of active ITP patients (Ma et al, 2012). Loss of RUNX1 in mouse BM cells was also shown to induce enhanced BMI1 expression (Nishimoto et al, 2011). Therefore, down-regulation of CDKN2A in cITP patients may be a consequence of BMI1 up-regulation by dysregulated RUNX1 mRNA expression. Alterations in the expression of RUNX1 itself may have similar effects. It is noteworthy that EWSR1 is inversely associated with CDKN2A expression (Cho et al, 2011), whereas EWSR1 exhibited increased expression (1.26-fold change, adjusted $P$ value $<10^{-16}$ ) in the present study. Overall, we suggest that a possible interplay between EWSR1, CDKN2A and RUNX in the context of cellular senescence may contribute to the pathophysiology of cITP.

In conclusion, we present numerous differentially expressed proteins in CITP that may be useful as a starting point for decoding the aetiology and pathogenesis of ITP. We also identified new factors and mechanisms, such as deregulated VEGFA signalling pathway, deregulated cellular senescence and apoptosis, and dysregulated expression of RUNX1 that appears to play a role in ITP. Future studies are required to further characterize these findings.

\section{Acknowledgments}

This study was funded by the German Research Foundation (DFG) (project number SA 405/3-1). M.E.F. acknowledges funding by the Portuguese Foundation for Science and Technology (FCT) (IF/00881/2013; UID/BIM/04773/2013 CBMR 1334).

\section{Authors' contributions}

G.B. conceived and designed the study, initiated the experiments, performed ELISA, LIA and Western blotting, contributed to the bioinformatic analysis of microarray data, interpreted the data and wrote the article; M.S.S.A. produced the microarrays and performed the antibody microarray experiments and quality control assessment of the microarray data; M.E.F. performed the bioinformatic analysis; D.H. performed the RUNX1 Western blot; F.R. performed RT-PCR experiments, V.S. contributed to the selection of patients and preparation of samples, and performed the RUNX3 Western blot; J.K.-M. obtained and provided clinically relevant patient data, contributed to the study design, and prepared serum samples. J.D.H and A.S. supervised and assisted with the design and coordination of the study. All authors read and edited the manuscript.

\section{Declaration of interest}

The authors declare no conflict of interest.

\section{Supporting Information}

Additional Supporting Information may be found in the online version of this article:

Table S1. *Platelet counts were set to $>250 \times 103 / \mu 1$ because only donors with platelet counts greater than $250 \times 103 / \mu \mathrm{l}$ are allowed to donate blood in our blood bank. The exact counts were not available in this study.

Table S2. Enrichment in the Gene Ontology (GO) categories of 161 differencial expressed protein in 679 distinct serum proteins as background dataset.

Table S3. List of 134 differencial expressed protein compared active cITP and remission cITP separately versus healthy donors. 


\section{References}

Adachi, M., Mita, S., Obana, M., Matsuoka, Y., Harada, K. \& Irimajiri, S. (1990) Thrombocytopenia subsequently develops systemic lupus erythematosus-can anti-SS-A antibody predict the next event? Japanese Journal of Medicine, 29, 481-486.

Alarcon-Riquelme, M.E. (2004) Role of RUNX in autoimmune diseases linking rheumatoid arthritis, psoriasis and lupus. Arthritis Research \& Therapy, 6, 169-173.

Alhamdani, M.S., Schroder, C. \& Hoheisel, J.D (2009) Oncoproteomic profiling with antibody microarrays. Genome Medicine, 1, 68

Alhamdani, M.S., Schroder, C. \& Hoheisel, J.D. (2010) Analysis conditions for proteomic profiling of mammalian tissue and cell extracts with antibody microarrays. Proteomics, 10, 32033207.

Alhamdani, M.S., Youns, M., Buchholz, M., Gress, T.M., Beckers, M.C., Marechal, D., Bauer, A. Schroder, C. \& Hoheisel, J.D. (2012) Immunoassay-based proteome profiling of 24 pancreatic cancer cell lines. Journal of Proteomics, 75, 3747-3759.

Andersson, P.O., Stockelberg, D., Jacobsson, S. \& Wadenvik, H. (2000) A transforming growth factor-betal-mediated bystander immune suppression could be associated with remission of chronic idiopathic thrombocytopenic purpura. Annals of Hematology, 79, 507-513.

Andersson, P.O., Olsson, A. \& Wadenvik, H. (2002) Reduced transforming growth factorbetal production by mononuclear cells from patients with active chronic idiopathic thrombocytopenic purpura. British Journal of Haematology, 116, 862-867.

Arandi, N., Mirshafiey, A., Jeddi-Tehrani, M., Shaghaghi, M., Sadeghi, B., Abolhassani, H., Sharifian, R.A., Rahiminejad, M.S. \& Aghamohammadi, A. (2014) Alteration in frequency and function of CD4(+)CD25(+)FOXP3 $(+)$ regulatory $\mathrm{T}$ cells in patients with immune thrombocytopenic purpura. Iranian Journal of Allergy, Asthma, and Immunology, 13, 85-92.

Ballem, P.J., Segal, G.M., Stratton, J.R., Gernsheimer, T., Adamson, J.W. \& Slichter, S.J. (1987) Mechanisms of thrombocytopenia in chronic autoimmune thrombocytopenic purpura. Evidence of both impaired platelet production and increased platelet clearance. The Journal of Clinical Investigation, 80, 33-40.

Balsalobre Aznar, J., Herraez Herrera, P., Porta Etessam, J., Torres Martin, C., Bermell Serrano, J.C., Nunez Lopez, R., Mateo Bernardo, I., Padrino Martinez, J.M. \& Morillas Lopez, L. (1999) Idiopathic thrombocytopenic purpura as first manifestation of systemic lupus erythematosus lupus. Anales de Medicina Interna, 16, 611614.

Besancenot, R., Chaligne, R., Tonetti, C., Pasquier, F., Marty, C., Lecluse, Y., Vainchenker, W., Constantinescu, S.N. \& Giraudier, S. (2010) A senescence-like cell-cycle arrest occurs during megakaryocytic maturation: implications for physiological and pathological megakaryocytic proliferation. PLoS Biology, 8, e1-11.

Casella, I., Feccia, T., Chelucci, C., Samoggia, P., Castelli, G., Guerriero, R., Parolini, I., Petrucci, E., Pelosi, E., Morsilli, O., Gabbianelli, M., Testa, U. \& Peschle, C. (2003) Autocrine-paracrine VEGF loops potentiate the maturation of megakaryocytic precursors through Flt1 receptor. Blood, 101, 1316-1323.

Chang-Lin, W.U., Jian-Cheng, X.U., Fang, L.I., Hong, X.I., Xue-Min, Z.H., Qun, C.H. \& XueWen, L.V. (2007) Polarization and apoptosis of $\mathrm{T}$ cell subsets in idiopathic thrombocytopenic purpura. International Journal of Laboratory Hematology, 29, 177-184.

Cho, J., Shen, H., Yu, H., Li, H., Cheng, T., Lee, S.B. \& Lee, B.C. (2011) Ewing sarcoma gene Ews regulates hematopoietic stem cell senescence. Blood, 117, 1156-1166.

Chomczynski, P. \& Sacchi, N. (1987) Single-step method of RNA isolation by acid guanidinium thiocyanate-phenol-chloroform extraction. Analytical Biochemistry, 162, 156-159.

Chousa, M., Ito, H., Saito, K., Takahashi, K., Takemura, M., Takahashi, T., Tomita, E. \& Seishima, M. (2008) The measurement of serum ceruloplasmin is useful for diagnostic differentiation of immune thrombocytopenic purpura. Clinica Chimica Acta, 389, 132-138.

Cines, D.B. \& Schreiber, A.D. (1979) Immune thrombocytopenia. Use of a Coombs antiglobulin test to detect IgG and C3 on platelets. New England Journal of Medicine, 300, 106-111.

Cines, D.B., Liebman, H. \& Stasi, R. (2009) Pathobiology of secondary immune thrombocytopenia. Seminars in Hematology, 46, S2-S14.

Emmerich, F., Bal, G., Barakat, A., Milz, J., Muhle, C., Martinez-Gamboa, L., Dorner, T. \& Salama, A. (2007) High-level serum B-cell activating factor and promoter polymorphisms in patients with idiopathic thrombocytopenic purpura. British Journal of Haematology, 136, 309-314.

Fahim, N.M. \& Monir, E. (2006) Functional role of $\mathrm{CD} 4+\mathrm{CD} 25+$ regulatory $\mathrm{T}$ cells and transforming growth factor-betal in childhood immune thrombocytopenic purpura. The Egyptian Journal of Immunology, 13, 173-187.

Fogarty, P.F. (2011) ITP: tolerance lost. Blood, 118, 6232-6234

Garcia-Suarez, J., Prieto, A., Reyes, E., Manzano, L., Merino, J.L. \& Alvarez-Mon, M. (1993) The clinical outcome of autoimmune thrombocytopenic purpura patients is related to their $\mathrm{T}$ cell immunodeficiency. British Journal of Haematology, 84, 464-470

Gerritsma, H., Schmid, A., Luethy, A.R., Leibundgut, K., Gugler, E., Wagner, H.P. \& Hirt, A. (1994) Megakaryocyte growth in vitro predicts outcome in idiopathic thrombocytopenic purpura. The American Journal of Pediatric Hematology/Oncology, 16, 194-199.

Gunsilius, E., Petzer, A.L. \& Gastl, G. (1999) Vascular endothelial growth factor platelet counts and renal cancer. Lancet, 353, 2247.
Guo, C., Chu, X., Shi, Y., He, W., Li, L., Wang, L., Wang, Y., Peng, J. \& Hou, M. (2007) Correction of Thl-dominant cytokine profiles by high-dose dexamethasone in patients with chronic idiopathic thrombocytopenic purpura. Journal of Clinical Immunology, 27, 557-562.

Han, X., Aslanian, A. \& Yates, J.R. 3rd (2008) Mass spectrometry for proteomics. Current Opinion in Chemical Biology, 12, 483-490.

Harrington, W.J., Minnich, V., Hollingsworth, J.W. \& Moore, C.V. (1951) Demonstration of thrombocytopenic factor in the blood of patients with thrombocytopenic purpura. Journal of Laboratory and Clinical Medicine, 38, 1-10.

Hazzan, R., Mukamel, M., Yacobovich, J., Yaniv, I. \& Tamary, H. (2006) Risk factors for future development of systemic lupus erythematosus in children with idiopathic thrombocytopenic purpura. Pediatric Blood \& Cancer, 47, 657-659.

Helms, C., Cao, L., Krueger, J.G., Wijsman, E.M., Chamian, F., Gordon, D., Heffernan, M., Daw, J.A., Robarge, J., Ott, J., Kwok, P.Y., Menter, A. \& Bowcock, A.M. (2003) A putative RUNX1 binding site variant between SLC9A3R1 and NAT9 is associated with susceptibility to psoriasis. Nature Genetics, 35, 349-356.

Hoheisel, J.D., Alhamdani, M.S. \& Schroder, C. (2013) Affinity-based microarrays for proteomic analysis of cancer tissues. Proteomics. Clinical applications, 7, 8-15.

Huang da, W., Sherman, B.T. \& Lempicki, R.A. (2009) Bioinformatics enrichment tools: path toward the comprehensive functional analysis of large gene lists. Nucleic Acids Research, 37, 1-13.

Jernas, M., Nookaew, I., Wadenvik, H. \& Olsson, B. (2013) Differential expression of T-cell genes in blood and bone marrow between ITP patients and controls. Thrombosis and Haemostasis, 109, 112-117.

Krauth, M.T., Puthenparambil, J. \& Lechner, K (2012) Paraneoplastic autoimmune thrombocytopenia in solid tumors. Critical Reviews in Oncology Hematology, 81, 75-81.

Kuwana, M., Kaburaki, J. \& Ikeda, Y. (1998) Autoreactive $\mathrm{T}$ cells to platelet GPIIb-IIIa in immune thrombocytopenic purpura. Role in production of anti-platelet autoantibody. The Journal of Clinical Investigation, 102, 1393-1402.

Li, X., Zhong, H., Bao, W., Boulad, N., Evangelista, J., Haider, M.A., Bussel, J. \& Yazdanbakhsh, K. (2012) Defective regulatory B-cell compartment in patients with immune thrombocytopenia. Blood, 120, 3318-3325.

Liebman, H.A. (2009) Recognizing and treating secondary immune thrombocytopenic purpura associated with lymphoproliferative disorders. Seminars in Hematology, 46, S33-S36.

Liebman, H.A. \& Stasi, R. (2007) Secondary immune thrombocytopenic purpura. Current Opinion in Hematology, 14, 557-573.

Liu, F., Wu, C., Yang, X., Xiao, H., Zhuo, X. Cheng, Z. \& Chen, Q. (2005) Polarization and apoptosis of $\mathrm{T}$ cell subsets in idiopathic thrombocytopenic purpura. Cellular \& Molecular Immunology, 2, 387-392. 


\section{G. Bal et al}

Lordier, L., Bluteau, D., Jalil, A., Legrand, C., Pan, J., Rameau, P., Jouni, D., Bluteau, O., Mercher, T., Leon, C., Gachet, C., Debili, N., Vainchenker, W., Raslova, H. \& Chang, Y. (2012) RUNX1-induced silencing of non-muscle myosin heavy chain IIB contributes to megakaryocyte polyploidization. Nature Communications, 3, 717.

Ma, D., Zhu, X., Zhao, P., Zhao, C., Li, X., Zhu, Y., Li, L., Sun, J., Peng, J., Ji, C. \& Hou, M. (2008) Profile of Th17 cytokines (IL-17, TGFbeta, IL-6) and Th1 cytokine (IFN-gamma) in patients with immune thrombocytopenic purpura. Annals of Hematology, 87, 899-904.

Ma, L., Zhou, Z., Zhang, D., Wang, H., Li, H., Xue, F. \& Yang, R. (2012) Bmi-1 regulates autoreactive CD4 $+\mathrm{T}$ cell survival in immune thrombocytopenia patients. Journal of Clinical Immunology, 32, 505-513.

Ma, L., Liang, Y., Fang, M., Guan, Y., Si, Y., Jiang, F. \& Wang, F. (2014) The cytokines (IFNgamma, IL-2, IL-4, IL-10, IL-17) and Treg cytokine (TGF-betal) levels in adults with immune thrombocytopenia. Die Pharmazie, 69, 694-697.

Makroo, R.N., Chowdhry, M., Mishra, M., Srivastava, P. \& Fauzdar, A. (2011) Octaploidy in idiopathic thrombocytopenic purpura. Indian Journal of Human Genetics, 17, 238-240.

Murakami, Y., Mizoguchi, F., Saito, T., Miyasaka, N. \& Kohsaka, H. (2012) p16(INK4a) exerts an anti-inflammatory effect through accelerated IRAK1 degradation in macrophages. Journal of Immunology, 189, 5066-5072.

Neunert, C., Lim, W., Crowther, M., Cohen, A., Solberg, L. Jr \& Crowther, M.A. (2011) The American Society of Hematology 2011 evidencebased practice guideline for immune thrombocytopenia. Blood, 117, 4190-4207.

Nishimoto, N., Arai, S., Ichikawa, M., Nakagawa, M., Goyama, S., Kumano, K., Takahashi, T., Kamikubo, Y., Imai, Y. \& Kurokawa, M. (2011) Loss of AML1/Runx1 accelerates the development of MLL-ENL leukemia through down-regulation of p19ARF. Blood, 118, 2541-2550.

Nobori, T., Miura, K., Wu, D.J., Lois, A., Takabayashi, K. \& Carson, D.A. (1994) Deletions of the cyclin-dependent kinase-4 inhibitor gene in multiple human cancers. Nature, 368, 753-756.

Norgaard, M., Jensen, A.O., Engebjerg, M.C., Farkas, D.K., Thomsen, R.W., Cha, S., Zhao, S. \& Sorensen, H.T. (2011) Long-term clinical outcomes of patients with primary chronic immune thrombocytopenia: a Danish population-based cohort study. Blood, 117, 3514-3520.

Ogawara, H., Handa, H., Morita, K., Hayakawa, M., Kojima, J., Amagai, H., Tsumita, Y., Kaneko, Y., Tsukamoto, N., Nojima, Y. \& Murakami, H. (2003) High Th1/Th2 ratio in patients with chronic idiopathic thrombocytopenic purpura. European Journal of Haematology, 71, 283-288.

Olsson, B., Andersson, P.O., Jernas, M., Jacobsson, S., Carlsson, B., Carlsson, L.M. \& Wadenvik, H.
(2003) T-cell-mediated cytotoxicity toward platelets in chronic idiopathic thrombocytopenic purpura. Nature Medicine, 9, 1123-1124.

Olsson, B., Andersson, P.O., Jacobsson, S., Carlsson, L. \& Wadenvik, H. (2005) Disturbed apoptosis of T-cells in patients with active idiopathic thrombocytopenic purpura. Thrombosis and Haemostasis, 93, 139-144.

Ono, M., Yaguchi, H., Ohkura, N., Kitabayashi, I., Nagamura, Y., Nomura, T., Miyachi, Y., Tsukada, T. \& Sakaguchi, S. (2007) Foxp3 controls regulatory $\mathrm{T}$-cell function by interacting with AML1/Runxl. Nature, 446, 685-689.

Otto, F., Lubbert, M. \& Stock, M. (2003) Upstream and downstream targets of RUNX proteins. Journal of Cellular Biochemistry, 89, 918.

Panitsas, F.P., Theodoropoulou, M., Kouraklis, A., Karakantza, M., Theodorou, G.L., Zoumbos, N.C., Maniatis, A. \& Mouzaki, A. (2004) Adult chronic idiopathic thrombocytopenic purpura (ITP) is the manifestation of a type-1 polarized immune response. Blood, 103, 2645-2647.

Parker, C.E. \& Borchers, C.H. (2014) Mass spectrometry based biomarker discovery, verification, and validation-quality assurance and control of protein biomarker assays. Molecular Oncology, 8, 840-858.

Rodeghiero, F., Stasi, R., Gernsheimer, T., Michel, M., Provan, D., Arnold, D.M., Bussel, J.B., Cines, D.B., Chong, B.H., Cooper, N., Godeau, B., Lechner, K., Mazzucconi, M.G., McMillan, R., Sanz, M.A., Imbach, P., Blanchette, V., Kuhne, T., Ruggeri, M. \& George, J.N. (2009) Standardization of terminology, definitions and outcome criteria in immune thrombocytopenic purpura of adults and children: report from an international working group. Blood, 113, 23862393.

Satoh, Y., Matsumura, I., Tanaka, H., Ezoe, S., Fukushima, K., Tokunaga, M., Yasumi, M., Shibayama, H., Mizuki, M., Era, T., Okuda, T. \& Kanakura, Y. (2008) AML1/RUNX1 works as a negative regulator of $\mathrm{c}-\mathrm{Mpl}$ in hematopoietic stem cells. Journal of Biological Chemistry, 283, 30045-30056.

Schroder, C., Jacob, A., Tonack, S., Radon, T.P., Sill, M., Zucknick, M., Ruffer, S., Costello, E., Neoptolemos, J.P., Crnogorac-Jurcevic, T., Bauer, A., Fellenberg, K. \& Hoheisel, J.D. (2010) Dual-color proteomic profiling of complex samples with a microarray of 810 cancer-related antibodies. Molecular \& Cellular Proteomics: MCP, 9, 1271-1280.

Semple, J.W. \& Freedman, J. (1991) Increased antiplatelet $\mathrm{T}$ helper lymphocyte reactivity in patients with autoimmune thrombocytopenia. Blood, 78, 2619-2625.

Semple, J.W., Milev, Y., Cosgrave, D., Mody, M., Hornstein, A., Blanchette, V. \& Freedman, J. (1996) Differences in serum cytokine levels in acute and chronic autoimmune thrombocytopenic purpura: relationship to platelet phenotype and antiplatelet T-cell reactivity. Blood, 87, 4245-4254.
Smyth, G.K. (2004) Linear models and empirical bayes methods for assessing differential expression in microarray experiments. Statistical Applications in Genetics and Molecular Biology, 3, e1-25. Article3.

Sood, R., Wong, W., Gotlib, J., Jeng, M. \& Zehnder, J.L. (2008) Gene expression and pathway analysis of immune thrombocytopenic purpura. British Journal of Haematology, 140, 99-103.

Stasi, R., Del Poeta, G., Stipa, E., Evangelista, M.L., Trawinska, M.M., Cooper, N. \& Amadori, S. (2007) Response to B-cell depleting therapy with rituximab reverts the abnormalities of $\mathrm{T}$-cell subsets in patients with idiopathic thrombocytopenic purpura. Blood, 110, 2924-2930.

Stasi, R., Sarpatwari, A., Segal, J.B., Osborn, J., Evangelista, M.L., Cooper, N., Provan, D., Newland, A., Amadori, S. \& Bussel, J.B. (2009) Effects of eradication of Helicobacter pylori infection in patients with immune thrombocytopenic purpura: a systematic review. Blood, 113, 1231-1240.

Stone, S., Jiang, P., Dayananth, P., Tavtigian, S.V., Katcher, H., Parry, D., Peters, G. \& Kamb, A. (1995) Complex structure and regulation of the P16 (MTS1) locus. Cancer Research, 55, 29882994.

Sullivan, P.S., Hanson, D.L., Chu, S.Y., Jones, J.L. \& Ciesielski, C.A. (1997) Surveillance for thrombocytopenia in persons infected with HIV: results from the multistate Adult and Adolescent Spectrum of Disease Project. Journal of Acquired Immune Deficiency Syndromes and Human Retrovirology, 14, 374-379.

Taniguchi, K., Kohsaka, H., Inoue, N., Terada, Y., Ito, H., Hirokawa, K. \& Miyasaka, N. (1999) Induction of the p16INK4a senescence gene as a new therapeutic strategy for the treatment of rheumatoid arthritis. Nature Medicine, 5, 760767.

Tokuhiro, S., Yamada, R., Chang, X., Suzuki, A., Kochi, Y., Sawada, T., Suzuki, M., Nagasaki, M., Ohtsuki, M., Ono, M., Furukawa, H., Nagashima, M., Yoshino, S., Mabuchi, A., Sekine, A., Saito, S., Takahashi, A., Tsunoda, T., Nakamura, Y. \& Yamamoto, K. (2003) An intronic SNP in a RUNX1 binding site of SLC22A4, encoding an organic cation transporter, is associated with rheumatoid arthritis. Nature Genetics, 35, 341348.

Ucar, C., Oren, H., Irken, G., Ates, H., Atabay, B., Turker, M., Vergin, C. \& Yaprak, I. (2003) Investigation of megakaryocyte apoptosis in children with acute and chronic idiopathic thrombocytopenic purpura. European Journal of Haematology, 70, 347-352.

Unal, I., Ceylan, C., Ozdemir, F., Turk, B.G., Kazandi, A.C. \& Kandiloglu, G. (2005) ITP as an initial manifestation of subacute cutaneous lupus erythematosus. Journal of Dermatology, 32, 727-730.

Wittmann-Liebold, B., Graack, H.R. \& Pohl, T. (2006) Two-dimensional gel electrophoresis as tool for proteomics studies in combination with 
protein identification by mass spectrometry. Proteomics, 6, 4688-4703.

Woolf, E., Xiao, C., Fainaru, O., Lotem, J., Rosen, D., Negreanu, V., Bernstein, Y., Goldenberg, D., Brenner, O., Berke, G., Levanon, D. \& Groner, Y. (2003) Runx3 and Runx1 are required for CD8 $\mathrm{T}$ cell development during thymopoiesis. Proceedings of the National Academy of Sciences of the United States of America, 100, 7731-7736.

Wu, K.H., Peng, C.T., Li, T.C., Wan, L., Tsai, C.H., Lan, S.J., Chang, M.C. \& Tsai, F.J. (2005) Interleukin 4, interleukin 6 and interleukin 10 polymorphisms in children with acute and chronic immune thrombocytopenic purpura. British Journal of Haematology, 128, 849-852.
Yamanaka, K. \& Takasaki, Y. (1993) Anti-PCNA antibody. Rinsho Byori. The Japanese Journal of Clinical Pathology, 41, 888-896.

Yehudai, D., Toubi, E., Shoenfeld, Y. \& Vadasz, Z. (2013) Autoimmunity and novel therapies in immune-mediated thrombocytopenia. Seminars in Hematology, 50, S100-S108.

Young, B., Levine, R.J., Salahuddin, S., Qian, C., Lim, K.H., Karumanchi, S.A. \& Rana, S. (2010) The use of angiogenic biomarkers to differentiate non-HELLP related thrombocytopenia from HELLP syndrome. The journal of Maternal-Fetal and Neonatal Medicine, 23, 366-370.

Yu, P., Qiu, S.W., Rao, Q., Lin, D., Xing, H.Y., Tang, K.J., Tian, Z., Wang, M. \& Wang, J.X.
(2012) Expression of c-MPL in leukemic stem cells from acute myeloid leukemia patients. Zhongguo Shi Yan Xue Ye Xue Za Zhi, 20, 10521055.

Zheng, C.X., Ji, Z.Q., Zhang, L.J., Wen, Q., Chen, L.H., Yu, J.F. \& Zheng, D. (2012) Proteomicsbased identification of haptoglobin as a favourable serum biomarker for predicting long-term response to splenectomy in patients with primary immune thrombocytopenia. Journal of Translational Medicine, 10, 208. 